the films with an $8 \mathrm{~nm}$ layer thickness exhibit a large pyroelectric coefficient between $10^{\circ} \mathrm{C}$ and $26^{\circ} \mathrm{C}$ with a maximum value of $4.1 \times 10^{-4} \mathrm{C} / \mathrm{m}^{2} \mathrm{~K}$ at $16^{\circ} \mathrm{C}$. This result demonstrates that the material is a good candidate for uncooled infrared focal plane array applications.

CORA LIND

\section{Broadband Frequency-Tunable Micromechanical Oscillator Promises to Extend the Applicability of Microelectro- mechanical Systems (MEMS)}

Scientists at the Cornell Center for Materials Research have developed a technique for varying the frequency of oscillations over a 300\% range in micronscale cantilever beams. These cantilevers, which are used as micromechanical oscillators, serve as the basic component in numerous microelectromechanical systems (MEMS) devices. When the oscillators are limited to operation at either a fixed resonant frequency or over a narrow frequency range, as, according to the researchers, has previously been the case, the applicability of these devices is restricted. Systems such as electromechanical filters, micromechanical spectrum analyzers, and magnetic resonance force microscopes (MFRM) may benefit from the researchers' work on broadband tunable microresonators.

As reported in the November 13 issue of Applied Physics Letters, M. Zalalutdinov, B. Ilic, D. Czaplewski, and co-workers employed a scanning tunneling microscope (STM) as a vibration actuator and a scanning electron microscope (SEM) as a motion detector in order to excite and detect oscillations in low-stress silicon nitride cantilevers $(200 \mu \mathrm{m} \times 20 \mu \mathrm{m} \times 0.6 \mu \mathrm{m})$. By applying a small ac voltage to a z-piezodrive, the tungsten STM tip, while in contact with the cantilever, was driven with $<0.1 \mathrm{~nm}$ amplitude in the direction perpendicular to the surface, thus causing it to behave as a point-like actuator. The resulting cantilever motion was detected by scanning the SEM electron beam across the cantilever edge and analyzing the secondary electron yield or video signal. By linearly scanning the electron beam over a distance greater than the motion of the cantilever edge, the resonant frequency of the cantilever oscillations was determined from the position of the peak in the resulting spectrum of the video signal.

Using the SEM, the resonant frequency was measured as a function of the STM tip distance from the cantilever end. The researchers report a continuously vari- able threefold increase in the resonant frequency as the STM was displaced toward the middle of the cantilever. The cantilever's deflection profile at a fixed tip position was also measured using the SEM. In both cases, the researchers found agreement between the experimental data and predictions obtained using the cantilever beam displacement equation.

"This method allows us to vary the cantilever over such a large frequency. Tunable micromechanical oscillators," said Zalalutdinov, "will provide the basis for the realization of solutions for diverse MEMS applications."

\section{STEFFEN K. KALDOR}

\section{High-Q Microcavity Based on Whispering Gallery Modes Constructed from Microsphere- Core-Shell Quantum Dot Structure}

A group of researchers at the University of Oregon in Eugene proposed and developed a quantum dot microcavity with extremely high $Q$ factors. They achieved two important parameters: small effective mode volume and long photon lifetime.

As reported in the November 1 issue of Optics Letters, the quantum dot microcavity was achieved by coupling core-shell CdSe-ZnS nanocrystals obtained by organometallic synthesis, with fused-silica microspheres (diameter $\sim 20 \mu \mathrm{m}$ to a few hundred microns) obtained by fusing a fiber tip with a $\mathrm{CO}_{2}$ laser. The CdSe$\mathrm{ZnS}$ nanocrystals used have a near-unity quantum yield at room temperature and a narrow linewidth-as determined by photoluminescence measurements (PL) on single quantum dots. To attach the nanocrystals to the fused-silica microsphere surface, the nanocrystals were suspended in a chloroform solution. After taking PL measurements of the composite nanocrystal-microsphere system, free spectral range of the whispering-gallery modes (WGM) of the microcavity was determined to be $0.7 \mathrm{~nm}$ for the 100- $\mu \mathrm{m}$ diameter of the sphere. Using a resonant light-scattering technique, the $Q$ factor was found to change its value from $1.6 \times 10^{6}$ to $1.6 \times 10^{8}$, corresponding to WGM linewidths of $4 \times 10^{-4} \mathrm{~nm}$ and $5 \times$ $10^{-6} \mathrm{~nm}$. The difference in the $Q$ values is due to the absorption of nanocrystals coupled with the relevant WGM. Furthermore, in order to increase the accuracy in $Q$ measurement, time-domain ringdown spectroscopy was used in two stages, before and after the sphere surface was reheated. Reheating the sphere surface increased the photon storage lifetime from $0.1 \mu$ s to $0.3 \mu \mathrm{s}$, corresponding to an increase in $Q$ from $2.4 \times 10^{8}$ to $7 \times 10^{8}$. The researchers concluded that the limiting factor in increasing $Q$ in the composite nanocrystal-microsphere system is the surface absorption on chloroform.

The research team anticipates that an even higher dipole coupling rate can be achieved using spheres with smaller diameters. According to the researchers, the extreme sensitivity of WGMs to the effects of single nanocrystals should open up a new avenue for probing dynamics, decoherence, and individual quantum transitions in a single quantum dot.

Iulia C. MunTELE

\section{Microprobing Silicon Surfaces Reveals Low-Resistance Surface Reconstructions}

An international team of scientists from the Technical University of Denmark and the School of Science of the University of Tokyo in Japan has discovered that using micro-four-point probes to measure the surface conductivity of structure results in a resistance of two orders of magnitude lower than that for $\mathrm{Si}(111)-\sqrt{3} \times \sqrt{3}-\mathrm{Ag}$ clean surfaces. The researchers attribute this difference to direct transport through surface states, an effect that cannot be observed with the conventional macroscopic four-point probes. Clean facets of many crystalline materials exhibit reconstructions of the outer atomic layers, which result in a new two-dimensional band structure at the surface. Although the dispersion of these bands can be measured by spectroscopic techniques, the characterization of charge transport in these surface states still remains a challenge.

As reported in the December 4 issue of Applied Physics Letters, the micro-four-point probes were prepared using silicon-based microfabrication technologies, following a procedure similar to that for atomic force microscope probes. The probes consist of four sharpened silicon oxide cantilevers coated with titanium, extending from a silicon chip. Electrode spacings of 8 and 20 $\mu \mathrm{m}$ were produced and used in these experiments. Once produced, the microscopic probes were integrated into a customized ultrahigh vacuum scanning electron microscope system, and manipulated with microslides for making contact with the analysis samples. The samples were $20 \times 3 \mathrm{~mm}^{2} n$-type $\mathrm{Si}(111)$, with a nominal resistivity of $10-100 \Omega \mathrm{cm}$. The sample surfaces were patterned with laser etching, in order to generate large step-free terraces, and then heated resistively at $1250^{\circ} \mathrm{C}$ in intervals of $10-60 \mathrm{~s}$ for a total of $3000 \mathrm{~s}$. The two surface reconstructions investigatedthe $\operatorname{Si}(111)-7 \times 7$ and $\mathrm{Si}(111)-\sqrt{3} \times \sqrt{3}-\mathrm{Ag}-$ 
should be conducting because both have a surface state at the Fermi energy.

The researchers concluded that a larger fraction of current runs in the space charge region for the microscopic probe, and that a larger fraction of current runs in the surface states. An estimate of the conductivity of the surface states can be deduced from the difference in conductance between the microprobe measurement and the theoretical prediction for band bending alone. The result is comparable more to conductivities for bulk materials such as bismuth. Since in this case the $\sqrt{3} \cap \sqrt{3}$ structure has no relationship with the metallic silver, it means that the conductivity result obtained is not due to a thin metallic film, but is an intrinsic property of the surface reconstruction.

Claudiu Muntele

\section{Holographic Two-Photon Polymerization Increases Speed of Switchable Gratings}

The fabrication of electrically switchable gratings consisting of periodic sheets of liquid crystal (LC) droplets and polymer has been reported by Senior Materials Research Engineer Timothy Bunning and his team from the Air Force Research Laboratory. These structures allow the rapid switching of information between two different states and thus possess great importance in the telecommunications, display, and computing industries. Gratings were fabricated by holographic two-photon induced photopolymerization (H-TPIP) of a homogeneous syrup mainly consisting of dipentaerythritol pentaacrylate (DPHPA) monomer and liquid crystal molecules. The resulting periodic struc-

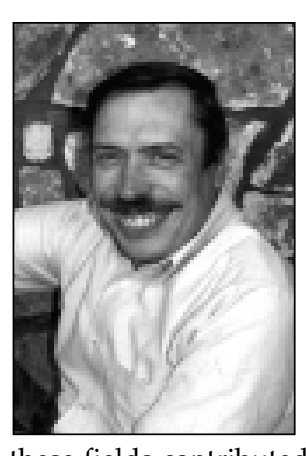

Alan Krauss passed away on June 26, 2000 after a courageous battle against cancer. He was a Senior Scientist and Group Leader at Argonne National Laboratory, where he had a distinguished career making major contributions to various interdisciplinary fields of research and technology development.

Alan was one of those scientists who embodied a deep knowledge of several fields of research, which included ion and plasma interaction with solids and gases, materials science (including bulk materials and thin films), and the invention and development of unique instrumentation which made a critical impact on the advancement of materials, surface and thin film science.

The experimental and theoretical work of Alan Krauss in these fields contributed to opening new avenues of research and to new technological developments. In this respect we can cite four major scientific and technological developments among Alan's contributions.

He helped develop the science and technology of unique alkali metal-based alloys, which made a major impact in the science and technology of fusion energy devices as well as on thin film-based cold cathodes for many important technologies such as field emission flat panel displays and high frequency devices. He was the co-developer of a unique microwave plasma technique to produce nanocrystalline diamond thin films. He made major contributions to the science and technology of multicomponent oxide thin films (e.g., high temperature superconductors and ferroelectrics), and he was a co-developer of a unique time-of-flight ion scattering and direct recoil- spectroscopy surface analysis technique, which for the first time can be used in relatively high-pressure environments.

Alan Krauss was not only a top scientist, but he also was one of those rare human beings who had an open mind and a gentle attitude toward his colleagues and other scientists. He always contributed to scientific discussions with constructive criticism and with challenging ideas. We as colleagues valued very much his positive approach to scientific discussions. Also, we could count on Alan when requesting from him contributions to conferences, invited papers, or articles for books. Alan was an excellent teacher. He directed numerous theses of graduate students from several universities in the United States. Those students went on to successful careers and in turn established scientific relationships and joint programs with Alan, which speaks for itself about the lasting legacy of his teaching.

Alan Krauss received several awards for his scientific accomplishments, and he was author or co-author of five patents and 11 patents pending. He published numerous papers and edited books that will provide continuous inspiration to colleagues and students for years to come. His scientific talent, dedication, warm personality, and friendship will be deeply missed.

ORLANDO AUCIELLO Argonne National Laboratory ture exhibits diffraction due to a periodic refractive index modulation; however, the intensity of the transmitted and diffracted beam can be varied by applying modest electrical fields to reorient the LC molecules, thereby changing the refractive index modulation. The H-TPIP process used by the AFRL team takes advantage of the nonlinear absorption of ultrafast laser pulses which can be raster scanned in three dimensions to write complex structures. The advantage of H-TPIP over conventional one-photon holographic processes is the much smaller local volume excited which allows sharper, more delineated local phase separation to occur.

As reported in the October issue of Chemistry of Materials, the grating was fabricated by irradiating a reactive mixture of the DPHPA monomer, bis(diphenylamino) diphenyl hexatriene initiator, Nvinyl pyrrolidinone reactive diluent, and nematic LC E7. The reactive mixture was sandwiched between two indium-tinoxide-coated glass slides at a thickness of $8 \mu \mathrm{m}$. The spatial and temporal overlap of two 90 -fs laser pulses $(950 \mu \mathrm{J}, 500 \mathrm{~Hz}$ repetition rate, $\lambda=800 \mathrm{~nm}$, spot size $7 \mathrm{~mm}^{2}$ ) induced photopolymerization through a free radical initiation process (H-TPIP). The free radicals were produced by the transfer of excited electrons from the initiator to the DPHPA anion following twophoton absorption. The polymerization was allowed to proceed for up to $60 \mathrm{~s}$.

Both optical and scanning electron microscope techniques were used to analyze the resulting polymerized structures. Polarized transmission microscopy of the grating revealed a polymer film with a grating spacing of about $3 \mu \mathrm{m}$. Low voltage scanning electron microscopic (LVSEM) analysis of the grating crosssections confirmed the period and revealed phase-separated, LC domains on the order of $20-200 \mathrm{~nm}$ in size. Only $25-30 \%$ of the grating period was composed of the LC-rich phase with the remainder of the structure being crosslinked polymer.

Previous work by the same researchers using one photon holographic polymerization produced a grating period composed of a much larger fraction of LC-rich domains. According to the researchers, the improved phase separation presented in this work is the result of the H-TPIP initiating a much smaller volume of the reactive mixture. Thus the distribution of initiating species is much different than in the one-photon holographic case. This skewed radical distribution modifies the resulting polymerization and phase separation dynamics relative to diffusion.

According to Bunning, these results 